TSUKUBA J. MATH.

Vol. 14 No. 1 (199()), 99-106

\title{
HARMONIC FOLIATIONS ON A COMPLEX PROJECTIVE SPACE
}

\author{
By \\ Tohru GoToH
}

\section{Introduction.}

In 1970, D. Ferus [6] gave an estimation on the codimension of a totally geodesic foliation on a sphere and a complex projective space, and successively P. Dombrowski [1] improved his results. Moreover, R. Escobales classified Riemannian foliations satisfying a certain condition on a sphere and a complex projective space in a series of his papers [2], [3], [4], [5].

On the other hand, F. Kamber and Ph. Tondeur [7], [8] studied the index of harmonic foliations with bundle-like metric on a sphere from a view point of harmonic mappings.

Recently, H. Nakagawa and R. Takagi [11] showed that any harmonic foliations on a compact Riemannian manifold of non-negative constant sectional curvature is totally geodesic if the normal plane field is minimal.

In this paper we will prove

THEOREM. Let $\mathbf{P}_{m}(\mathbf{C})$ be a complex projective space of complex dimension $m$ with the metric of constant holomorphic sectional curvature. If $\mathcal{I}$ is a harmonic foliation on $\mathbf{P}_{m}(\mathbf{C})$ snch that the normal plane field is minimal, then $\mathscr{I}$ is totally geodesic.

I am grateful to professor Ryoichi Takagi for his kind guidance and constant encouragement.

\section{Preliminaries.}

We first establish some basic notations and formulas in the theory of foliated Riemannian manifolds. For details, see [9], [10], [11], [13].

Let $(M, g)$ be an $n$-dimentional Riemannian manifold and $\mathscr{I}$ a foliation with codimension $q$ on $M$. Considering $\mathscr{T}$ as an $(n-q)$-dimensional integrable distribution on $M$, we denote the orthogonal distribution of $\mathscr{F}$ by $\mathscr{F}^{\perp}$, which is called the normal plane field.

Rceeived February 16, 1989. Revised May 22, 1989. 
Therefore if we denote the space of vector fields on $M$ by $\mathscr{X}(M)$, each $X \in \mathfrak{X}(M)$ can be decomposed as $X=X^{\prime}+X^{\prime \prime}$, where $X_{x}^{\prime} \in \mathscr{F}_{x}$ and $X_{x}^{\prime \prime} \in \mathscr{F}_{x}^{\frac{1}{x}}$ for each $x \in M$. Then two tensor fields $A$ and $h$ of type (1.2) on $M$ are defined by

$$
\begin{aligned}
& A(X, Y)=-\left(\nabla_{Y^{\prime \prime}} X^{\prime \prime}\right)^{\prime}, \\
& h(X, Y)=\left(\nabla_{Y^{\prime}} X^{\prime}\right)^{\prime \prime}, \quad X, Y \in \mathfrak{X}(M) .
\end{aligned}
$$

The ristriction of $h$ to each leaf of $\mathscr{I}$ is so-called the second fundamental form of the leaf.

Now, according to [11], we express them with respect to locally defined orthonormal frame field.

As for the range of indices the following convention will be used throughout this paper unless otherwise stated:

$$
\begin{gathered}
A, B, C, \cdots=1,2,3, \cdots, n \\
i, j, k, \cdots=1,2,3, \cdots, p \\
\alpha, \beta, \gamma, \cdots=p+1, \cdots, n,
\end{gathered}
$$

where $p=n-q$ is the dimension of $\mathscr{F}$.

Let $\left\{e_{1}, e_{2}, \cdots, e_{n}\right\}$ be a locally defined orthonormal frame field of $M$ such that $e_{1}, e_{2}, \cdots, e_{p}$ are always tangent to $\mathscr{F}$. Denote its dual by $\left\{\omega_{1}, \omega_{2}, \cdots, \omega_{n}\right\}$.

The Riemannian connection form $\left\{\omega_{A B}\right\}$ with respect to $\left\{\omega_{A}\right\}$ are defined by the followings:

$$
\begin{aligned}
& \omega_{A B}+\omega_{B A}=0, \\
& d \omega_{A}+\Sigma \omega_{A B} \wedge \omega_{B}=0 .
\end{aligned}
$$

A relation between $\omega_{A B}$ and $\nabla$ is given by

$$
\nabla_{e_{A}} e_{B}=\Sigma \omega_{C B}\left(e_{A}\right) e_{C} .
$$

Then the components $h_{B C}^{A}$ (resp. $A_{C D}^{B}$ ) of $h$ (resp. $A$ ) with respect to $\left\{e_{A}\right\}$ and $\left\{\omega_{A}\right\}$ are given by

$$
h_{i j}^{\alpha}=\omega_{\alpha i}\left(e_{j}\right) \quad\left(\text { resp. } A_{\alpha \beta}^{i}=\omega_{\alpha i}\left(e_{\beta}\right)\right),
$$

and any other components vanish.

Since the distribution $\omega_{\alpha}=0$ is integrable,

$$
h_{i j}^{\alpha}=h_{j i}^{\alpha} .
$$

The foliation $\mathscr{I}$ is said to be harmonic or minimal (resp. totally geodesic) provided that $\sum h_{i i}^{\alpha}=0$ (resp. $h_{i j}^{\alpha}=0$ ), and owing to [9], [13], the normal plane field $\mathscr{F}^{\perp}$ is said to be minimal provided that $\Sigma A_{\alpha \alpha}^{i}=0$. 
A necessary and sufficient condition for the distribution $\omega_{i}=0$ to be integrable is $A_{\alpha \beta}^{i}=A_{\beta \alpha}^{i}$. On the contrary, the Riemannian metric $g$ is bundle-like if and only if

$$
A_{\alpha \beta}^{i}=-A_{\beta \alpha}^{i} \text {. }
$$

The curvature form $\Omega=\left(\Omega_{A B}\right)$ of $M$ is defined by

$$
\Omega_{A B}=d \omega_{A B}+\sum \omega_{A C} \wedge \omega_{C B},
$$

and we define its components $R_{A B C D}$ by

$$
\Omega_{A B}=-(1 / 2) \sum R_{A B C D} \omega_{C} \wedge \omega_{D}, \quad R_{A B C D}+R_{A B D C}=0 .
$$

Then the equalities $R_{A B C D}=-R_{B A C D}=R_{C D A B}$ hold.

Now for an $(r, s)$-tensor field $T=\left(T_{\left.B_{1} B_{2} \cdots B_{s}^{A}\right)}^{A_{1} A_{2}}\right.$ on $M$, we define the coveriant derivative $\nabla T=\left(T_{B_{1} B_{2} \cdots B_{s}^{A} D}^{A_{2}}\right)$ by

$$
\begin{aligned}
& \Sigma T_{B_{1} B_{2} \cdots B_{s} C}^{A_{1} A_{2} \cdots \omega_{C}}=d T_{B_{1} B_{2}}^{A_{1} A_{2} \cdots B_{s}}
\end{aligned}
$$

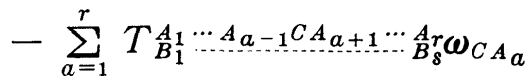

$$
\begin{aligned}
& -\sum_{b=1}^{s} T_{B_{1} \cdots B_{b-1} C B_{b+1} \cdots B_{s}^{A}}^{A_{1}} \omega_{C B_{b}} .
\end{aligned}
$$

Then we have followings $([11])$ :

$$
\begin{gathered}
h_{i j k}^{\alpha}-h_{i k j}^{\alpha}=R_{\alpha i j k}, \\
h_{i j \beta}^{\alpha}-A_{\alpha \beta j}^{i}-\sum h_{i k}^{\alpha} h_{k j}^{\beta}-\sum A_{\alpha \gamma}^{i} A_{\gamma \beta}^{j}=R_{\alpha i j \beta}, \\
A_{\alpha \beta \gamma}^{i}-A_{\alpha \gamma \beta}^{i}+\sum h_{i j}^{\alpha}\left(A_{\beta \gamma}^{j}-A_{\gamma \beta}^{j}\right)=-R_{\alpha i \gamma \beta} .
\end{gathered}
$$

From now on, we consider the case where $M$ is the complex projective space $\mathbf{P}_{m}(\mathbf{C})$ of complex dimension $m(=n / 2)$ with the metric of constant holomorphic sectional curvature $4 c$.

Let $J$ denote the complex structure of $\mathbf{P}_{m}(\mathbf{C})$ and put $J\left(e_{A}\right)=\sum J_{B A}\left(e_{B}\right)$. Then $\left(J_{A B}\right)$ satisfies

$$
\begin{aligned}
& J_{A B}+J_{B A}=0, \\
& \Sigma J_{A C} J_{C B}=-\delta_{A B}, \\
& d J_{A B}=\Sigma\left(J_{A C} \omega_{C B}-J_{B C} \omega_{C A}\right) .
\end{aligned}
$$

The last equation means that $\nabla J=0$. Moreover the curvature form $\Omega=\left(\Omega_{A B}\right)$ and its components $R_{A B C D}$ defined by (1.7) and (1.8) respectively are given by

$$
\begin{aligned}
& \Omega_{A B}=c \omega_{A} \wedge \omega_{B}+c \sum\left(J_{A C} J_{B D}+J_{A B} J_{C D}\right) \omega_{C} \wedge \omega_{D}, \\
& R_{A B C D}=c\left(\delta_{A D} \delta_{B C}-\delta_{A C} \delta_{B D}\right)+c\left(J_{A D} J_{B C}-J_{A C} J_{B D}-2 J_{A B} J_{C D}\right) .
\end{aligned}
$$


Therefore we obtain

$$
R_{A B C D E}=0 \text {. }
$$

\section{Proof of the main theorem.}

In this section we give the proof of our main theorem. In the case where $p=1$, any harmanic foliation is necessarily totally geodesic. Therefore we may assume $p \geqq 2$.

Consider the global vector field $v=\sum v_{A} e_{A}$ on $\mathbf{P}_{m}(\mathbf{C})$ defined by

$$
v_{k}=\sum h_{i j}^{\alpha} h_{i j k}^{\alpha}, \quad v_{\alpha}=0 .
$$

We first calculate the divergence $\delta v$ of $v$.

In general H. Nakagawa and R. Takagi showed the following lemma $([11])$ :

LEMMA 2.1. Let $(M, g, \mathscr{F})$ be a faliated Riemannian manifold and $v$ a vector field on $M$ defined above. Then

(1) the divergence $\delta v$ of $v$ is given by

$$
\begin{aligned}
\delta v= & \sum v_{i} A_{\alpha \alpha}^{i}+\sum h_{i j k}^{\alpha} h_{i j k}^{\alpha}+\sum h_{i j}^{\alpha} R_{\alpha i j k k} \\
& +\sum h_{i j}^{\alpha} R_{\alpha k i k j}+\sum h_{i j}^{\alpha} h_{k k}^{\beta} h_{i j \beta}^{\alpha}+\sum h_{i j}^{\alpha} h_{k k i j}^{\alpha} \\
& +\sum\left(h_{i k}^{\beta} R_{\alpha \beta j k}+h_{l k}^{\alpha} R_{i l j k}+h_{i l}^{\alpha} h_{k l j k}\right) h_{i j}^{\alpha} \\
& +\sum h_{i j}^{\alpha} h_{l k}^{\alpha} h_{i j}^{\beta} h_{l k}^{\beta}+2 \sum h_{i j}^{\alpha} h_{i k}^{\beta} h_{j l}^{\alpha} h_{l k}^{\beta},
\end{aligned}
$$

and

(2) if the foliation $\mathscr{I}$ is harmonic,

$$
\sum h_{i j k}^{\alpha}=-2 \sum h_{i j}^{\beta} h_{i l}^{\alpha} h_{l k}^{\beta} .
$$

Therefore if the foliation $\mathscr{F}$ is harmonic and the normal plane field $\mathscr{F}^{\perp}$ minimal, we obtain

$$
\begin{aligned}
\delta v= & \sum h_{i j k}^{\alpha} h_{i j k}^{\alpha}+\sum h_{i j}^{\alpha} h_{l k}^{\alpha} h_{i j}^{\beta} h_{l k}^{\beta} \\
& +2 \sum \operatorname{Tr}\left(H^{\alpha} H^{\alpha} H^{\beta} H^{\beta}-H^{\alpha} H^{\beta} H^{\alpha} H^{\beta}\right) \\
& +\sum\left(h_{i k}^{\beta} R_{\alpha \beta j k}+h_{l k}^{\alpha} R_{i l j k}+h_{i l}^{\alpha} R_{k l j k}\right) h_{i j}^{\alpha},
\end{aligned}
$$

where $H^{\alpha}$ denotes the $p \times p$ matrix $\left(h_{i j}^{\alpha}\right)$.

The essential part of the proof is to show that $\delta v$ is non-negative on $\mathbf{P}_{m}(\mathbf{C})$. For it, putting

$$
X=\sum\left(h_{i k}^{\beta} R_{\alpha \beta j k}+h_{l k}^{\alpha} R_{i l j k}+h_{i l}^{\alpha} R_{k j l k}\right) h_{i j}^{\alpha},
$$

we have only to show $X \geqq 0$, since 


$$
\operatorname{Tr}\left(H^{\alpha} H^{\alpha} H^{\beta} H^{\beta}-H^{\alpha} H^{\beta} H^{\alpha} H^{\beta}\right) \geqq 0 \text { holds }([11]) .
$$

For simplicity we put

$$
\xi_{i j k}=\sum h_{i j}^{\alpha} J_{\alpha k}, \quad \eta_{i \beta}^{\alpha}=\sum h_{i j}^{\alpha} J_{\beta j}, \quad \mu_{i j}^{\alpha}=\Sigma h_{i k}^{\alpha} J_{k j} .
$$

Then from (1.13), (1.16), we have

$$
\begin{aligned}
X= & \sum_{\alpha, i, j} c p\left(h_{i j}^{\alpha}\right)^{2}+c Y \\
& +3 c \sum_{\alpha}\left\{2 \sum_{i}\left(\mu_{i i}^{\alpha}\right)^{2}+\sum_{i<k}\left(\mu_{i k}^{\alpha}+\mu_{k i}^{\alpha}\right)^{2}\right\},
\end{aligned}
$$

where we put

$$
Y=\sum h_{i j}^{\alpha} h_{i k}^{\beta}\left(J_{\alpha k} J_{\beta j}-J_{\alpha j} J_{\beta k}-2 J_{\alpha \beta} J_{j k}\right) .
$$

Next lemma gives the key inequality.

LEMMA 2.2. For the $Y$ above, the following inequality holds:

$$
\begin{aligned}
Y \geqq & -\left\{\left((p-1)^{2}+1\right) /(p-1)\right\} \sum_{i, j, \alpha}\left(h_{i j}^{\alpha}\right)^{2} \\
& +(p-2) \sum_{i} \sum_{j \neq k}\left(\xi_{i j k}\right)^{2}+\sum_{i} \sum_{j<k}\left(\xi_{i j k}+\xi_{i k j}\right)^{2} \\
& +\sum_{i} \sum_{j<k}\left(\xi_{i j j}-\xi_{i k k}\right)^{2}+(p-1)^{-1} \sum_{i, \alpha, \beta}\left(\eta_{i \alpha}^{\beta}\right)^{2} .
\end{aligned}
$$

PROOF of lemma 2.2. For any real number $t \neq 0$, an inequality $\left(t \sum h_{i j}^{\alpha} J_{\alpha \beta}\right.$ $\left.t^{-1} \sum h_{i k}^{\beta} J_{j k}\right)^{2} \geqq 0$ holds, which implies

$$
-2 \sum h_{i j}^{\alpha} h_{i k}^{\beta} J_{\alpha \beta} J_{j k} \geqq-t^{2} \sum h_{i j}^{\alpha} J_{\alpha \beta} h_{i j}^{\gamma} J_{\gamma \beta}-t^{-2} \sum h_{i k}^{\beta} J_{j k} h_{i l}^{\beta} J_{j l} .
$$

By (1.10), the right hand side of this equation is equal to

$$
\begin{aligned}
& =-t^{2} \sum h_{i j}^{\alpha} h_{i j}^{\gamma}\left(-\sum J_{\alpha k} J_{\gamma k}+\delta_{\alpha \gamma}\right)-t^{-2} \sum h_{i k}^{\beta} h_{i l}^{\beta}\left(-J_{\alpha k} J_{\alpha l}+\delta_{k l}\right) \\
& =-\left(t^{2}+t^{-2}\right) \sum_{i, j, \alpha}\left(h_{i j}^{\alpha}\right)^{2}+t^{2} \sum_{i, j, k}\left(\xi_{i j k}\right)^{2}+t^{-2} \sum_{i, \alpha_{,} \beta}\left(\eta_{i \alpha}^{\beta}\right)^{2} .
\end{aligned}
$$

Therefore, putting $t=\sqrt{p-1}$, we obtain

$$
\begin{aligned}
Y+ & \left\{\left((p-1)^{2}+1\right) /(p-1)\right\} \sum_{i, j, \alpha}\left(h_{i j}^{\alpha}\right)^{2} \\
\geqq & \sum_{i, j, k} \xi_{i j k} \xi_{i k j}-\sum_{i, j, k} \xi_{i j j} \xi_{i k k}+(p-1) \sum_{i, j, k}\left(\xi_{i j k}\right)^{2}+(p-1)^{-1} \sum_{i, \alpha, \beta}\left(\eta_{i \alpha}^{\beta}\right)^{2} \\
= & \sum_{i, j}\left(\xi_{i j j}\right)^{2}+2 \sum_{i} \sum_{j<k} \xi_{i j k} \xi_{i k j}-\sum_{i, j}\left(\xi_{i j j}\right)^{2}-2 \sum_{i} \sum_{j<k} \xi_{i j j} \xi_{i k k} \\
& +(p-1) \sum_{i, j}\left(\xi_{i j j}\right)^{2}+(p-1) \sum_{i} \sum_{j \neq k}\left(\xi_{i j k}\right)^{2}+(p-1)^{-1} \sum_{i, \alpha, \beta}\left(\eta_{i \alpha}^{\beta}\right)^{2} \\
= & (p-2) \sum_{i} \sum_{j \neq k}\left(\xi_{i j k}\right)^{2}+\sum_{i} \sum_{j<k}\left(\xi_{i j k}+\xi_{i k j}\right)^{2}+\sum_{i} \sum_{j<k}\left(\xi_{i j j}-\xi_{i k k}\right)^{2}+(p-1)^{-1} \sum_{i, \alpha, \beta}\left(\eta_{i \alpha}^{\beta}\right)^{2},
\end{aligned}
$$


which is the required inequality.

(q.e.d.)

We are now in a position to complete the proof of the theorem. Owing to lemma 2.2 , (2.1) and (2.2), we obtain

$$
\begin{aligned}
\delta v \geqq & \sum_{i, j, k, \alpha}\left(h_{i j k}^{\alpha}\right)^{2}+\sum_{i, j, k, l, \alpha}\left(\sum h_{i j}^{\alpha} h_{l k}^{\alpha}\right)^{2}+2 \sum_{\alpha, \beta} \operatorname{Tr}\left(H^{\alpha} H^{\alpha} H^{\beta} H^{\beta}-H^{\alpha} H^{\beta} H^{\alpha} H^{\beta}\right) \\
& +c\{(p-2) /(p-1)\} \sum_{i, j, \alpha}\left(h_{i j}^{\alpha}\right)^{2}+c(p-2) \sum_{i} \sum_{j \neq k}\left(\xi_{i j k}\right)^{2}+c \sum_{i} \sum_{j<k}\left(\xi_{i j k}+\xi_{i k j}\right)^{2} \\
& +c \sum_{i} \sum_{j<k}\left(\xi_{i j j}-\xi_{i k k}\right)^{2}+\{c /(p-1)\} \sum_{i, \alpha, \beta}\left(\eta_{i \alpha}^{\beta}\right)^{2} \\
& +3 c \sum_{\alpha}\left\{2 \sum_{i}\left(\mu_{i i}^{\alpha}\right)^{2}+\sum_{i<k, \alpha}\left(\mu_{i k}^{\alpha}+\mu_{k i}^{\alpha}\right)^{2}\right\} \geqq 0,
\end{aligned}
$$

since $p \geqq 2$ by assumption.

Since $\mathbf{P}_{m}(\mathbf{C})$ is orientable and compact, we have

$$
\int_{\mathbf{P}_{m}(\mathbf{C})} \delta v * 1=0,
$$

where $* 1$ denotes the volume element of $\mathbf{P}_{m}(\mathbf{C})$. This together with the above inequality shows

$$
\sum h_{i j}^{\alpha} h_{k l}^{\alpha}=0, \quad \text { and so } h_{i j}^{\alpha}=0 .
$$

The theorem is now completely proved.

Next corollary is now obvious :

Corollary. Let $\mathbf{P}_{m}(\mathbf{C})$ be the complex projective space of complex dimension $m$ with the metric of constant holomorphic sectional curvature. Let $\mathcal{I}$ be a harmonic foliation for which the metric is bundle-like. Then the foliation $\mathcal{F}$ is totally geodesic.

\section{Some other results and remarks.}

In this section the preceding notations are kept.

We call a foliation on $\mathbf{P}_{m}(\mathbf{C})$ Kähler (resp. totally real) if $J_{\alpha i}=0$ (resp. $\left.J_{i j}=0\right)$ at each point.

Let $\mathscr{F}$ be a totally geodesic foliation on $\mathbf{P}_{m}(\mathbf{C})$. Then from (1.10) and (1.16) we obtain

$$
J_{\alpha k} J_{i j}-J_{\alpha j} J_{i k}-2 J_{\alpha i} J_{j k}=0 .
$$

Therefore

$$
0=\Sigma\left(J_{\alpha k} J_{i j}-J_{\alpha j} J_{i k}-2 J_{\alpha i} J_{j k}\right) J_{\alpha j} J_{i k}
$$




$$
=-\sum_{\alpha, i}\left(\sum_{j} J_{\alpha j} J_{i j}\right)^{2}-\sum_{i, j, k, \alpha}\left(J_{\alpha j} J_{i k}\right)^{2}
$$

which implies

$$
J_{\alpha j}=0 \text { or } J_{i k}=0 \text { at each point. }
$$

Proposition 3.1. Let $\mathscr{I}$ be a totally geodesic foliation on $\mathbf{P}_{m}(\mathbf{C})$. Then $\mathscr{T}$ is Kähler or totally real.

PROOF.

Set $K=\left\{x \in \mathbf{P}_{m}(\mathbf{C}) \mid \mathscr{T}\right.$ is Kähler at $\left.x\right\}$ and $T=\left\{x \in \mathbf{P}_{m}(\mathbf{C}) \mid \mathscr{F}\right.$ is totally real at $\left.x\right\}$. Then (3.1) implies the followings :

(a) $K$ and $T$ are open in $\mathbf{P}_{m}(\mathbf{C})$,

(b) $K \cap T=\varnothing$,

(c) $K \cup T=\mathbf{P}_{m}(\mathbf{C})$.

These (a), (b), (c) and connectedness of $\mathbf{P}_{m}(\mathbf{C})$ show the assertion. (q.e.d.)

REMARK 1. There is a well-known example of a foliation on a complex projective space which is induced by the fiber bundle

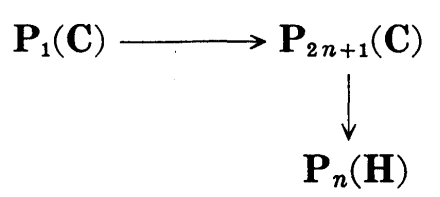

where $\mathbf{P}_{n}(\mathbf{H})$ denotes the quaternionic projective $n$-space.

R. Escobales [5] has proved that the above example is the only non-trivial Riemannian foliation on $\mathbf{P}_{n}(\mathbf{C})$ by $\mathbf{P}_{k}(\mathbf{C})$ by making use of his results [3], [4] and Ucci's result [15].

REMARK 2. The above example is totally geodesic and Kähler. The auther does not know examples of totally geodesic and totally real foliations on a complex projective space.

Does there exist a totally geodesic foliation on a complex projective space which is totally real?

This question seems to be of interest.

\section{References}

[1] Dombrowski, P., Jacobi fields, totally geodesic foliations, and geodesic differential forms, Resultate der Math. 1 (1978), 156-194. 
[2] Ecobales, R. Jr., Riemannian submersions with totally geodesic fibers, J. Differential Geom. 10 (1975), 253-276.

[3] Escobales, R. Jr., Riemannian submersions from complex projective space, J. Differential Geom. 13 (1978), 93-107.

[4] Escobales, R. Jr., Sufficient conditions for a bundle like foliation to admit a Riemannian submersion onto its leaf space, Proc. Amer. Soc. 84 (1982), 280-284.

[5] Escobales, R. Jr., Riemannian foliations of the rank one symmetric space, Proc. Amer. Math. Soc. 95 (1985), 495-498.

[6] Ferus, D., Totally geodesic foliations, Math. Ann. 188 (1970), 313-316.

[7] Kamber, F.W. and Tondeur, Ph., Infinitesimal automorphisms and second variation of the energy for harmonic foliation, Tôhoku Math. J. 34 (1982), 525-538.

[8] Kamber, F.W. and Tondeur, Ph., The index of harmonic foliations on spheres, Tarns. Amer. Math. Soc. 275 (1983), 257-263.

[9] Kitahara, H., Differential geometry of Riemannian foliations, Lecture notes, Kyungpook National Univ. 1986.

[10] Molino, P., Feuilletagesriemannien, Lecture notes, Université des Sciences et Techniques du Languedoc, 1982-1983.

[11] Nakagawa, H. and Takagi, R., Harmonic foliations on a compact Riemannian manifold of non-negative constant curvature, Tôhoku Math. J. 40 (1988), 465-471.

[12] O'Neill, B., The fundamental equations of a submersion, Michigan Math. J. 13 (1966), 459-469.

[13] Reinhart, B., Differential geometry of foliations, Springer-Verlag, Berlin, Heidelberg, New York, 1983.

[14] Tondeur, Ph., Foliations on Riemannian Manifolds, Universitext, Springer-Verlag, 1988.

[15] Ucci, J., On the non-existence of Riemannian submersions from $\boldsymbol{C P}(7)$ and $\boldsymbol{C P}(3)$, Proc. Amer. Math. Soc. 88 (1983), 698-700.

Department of Mathematics

Faculty of Mathematics and Physical Sciences

Graduate School of Science and Technology

Chiba University

Chiba, 260

Japan 